\title{
Signaling Alternatives in a Wireless ATM Network
}

\author{
Bora A. Akyol, Student Member, IEEE, and Donald C. Cox, Fellow, IEEE
}

\begin{abstract}
The world of wireless telecommunications is rapidly changing. The capabilities of wireless networks are improving at a steady pace. This paper presents two possible protocols for implementing mobility for wireless users in an asynchronous transfer mode (ATM) network. The vision of the authors is of one "wireless ATM telecommunications network" that is capable of supporting a variety of today's applications with room to grow for advanced applications of the future. We first visit database architectures that can support mobility in a wireless ATM network. We then discuss one of two signaling architecture alternatives, the "overlay signaling," for overlay support of mobile users in the ATM-based wireless telecommunications network. "Overlay signaling" aims at minimizing the modification needed to the existing ATM protocols. We then describe a native "migratory signaling" approach that further integrates wireless and wireline users into one global wireless ATM network at the expense of requiring some modifications to the existing ATM protocols. A performance analysis of the proposed signaling architecture alternatives is also presented. We conclude by pointing out some challenges in merging ATM with wireless telecommunications.
\end{abstract}

Index Terms - Handoff in wireless ATM networks, mobility management in ATM networks, signaling protocols, wireless ATM networking.

\section{INTRODUCTION}

I $\mathrm{N}$ THIS paper, we focus on the signaling aspects of designing an asynchronous transfer mode (ATM) based wireless personal communications network (PCN). Two approaches that may be used for devising a signaling protocol for such a network are identified. The first approach, called the "overlay" approach, keeps the existing ATM signaling protocol intact, and functions as an overlay network. This first approach is described in this paper and in [1]. The second approach, which is referred to as the "migratory approach," modifies the existing ATM signaling protocols to accommodate mobile users together with the fixed users and aims to minimize the overhead incurred in supporting mobile users. The migratory approach to wireless ATM signaling is the second part of this paper. These two approaches may be used together as a migratory path to a unique global wireless ATM network. We first discuss the signaling procedures used in the overlay and migratory approaches to support users in the wireless ATM network. Then, we give quantitative results on the overhead incurred by the two alternative signaling protocols. We then note some of the challenges in incorporating mobility in an ATM

Manuscript received February 1, 1996; revised June 1996. This work was supported by by Motorola Inc., Schaumburg, IL, and Pacific Bell, San Ramon, CA.

The authors are with the Department of Electrical Engineering, Stanford University, Stanford, CA 94305 USA (email: akyol@stanford.edu).

Publisher Item Identifier S 0733-8716(97)00051-6.

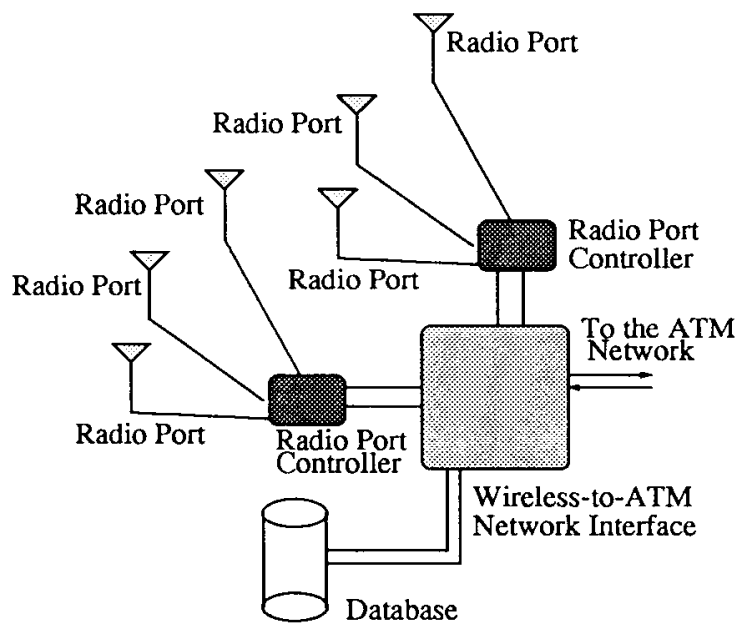

Fig. 1. A zone in the wireless ATM PCN.

network. Before discussing these topics, we digress briefly to consider a possible system architecture for supporting mobility in an ATM network.

\section{The ARChiteCtURE TO SUPPORT MOBILITY IN AN ATM NETWORK}

Mobility is a key advantage of a wireless network. In order to provide mobility, the wireless network must keep track of the location of its users. In a network where users are globally mobile, the tracking of users is one of the major functions of the wireless network. A database system is used to support the tracking process. The two-tier database architecture used in this research is advantageous in a geographically partitioned network such as a personal communication services (PCS's) network. We assume that the wireless network service area is partitioned into "zones." Each zone consists of a set of radio ports, the radio port controllers, and the ATM network interface equipment that interconnects these radio ports and provides fixed network access (see Fig. 1). Two types of fixed network connections exist in a zone. The first is the ATM network that interconnects the zones in the wireless network. The second type of connection is the connection between the radio ports and the radio port controllers. This second type of connection is not within the scope of this paper and is not discussed in the text. Hence, in this paper, we assume that the ports and the port controllers are interconnected by an optimized communications link.

There is also a database associated with every zone. This database is partitioned into two segments. One segment is 
reserved for the users that are permanently registered in that zone, i.e., the "home" segment. The second segment is for the users are that are visiting the zone. The network databases do not keep track of the radio ports that connect the users to the network. The port controller keeps track of radio port and active user information.

The user identification numbers determine the location of user's permanent registry or home database. The nearest database is the first place to be searched in attempting to locate user information. If the desired information is not found at the nearest database then the permanent home database for that user is queried. We will refer to this architecture as the "two-tier database architecture." The European GSM and U.S. IS-41 digital cellular standard of "home location register/visiting location register" is one example of a twotier database architecture [8], [9]. One important advantage of a two-tier architecture as described above is the two-step deterministic search where a user's profile is retrieved in at most two database look-ups.

An alternative to a two-tier database architecture is a hierarchical structure which takes advantage of the locality and motion patterns of users and organizes the distribution of data accordingly. The main difference in terms of user identification numbers between a hierarchical scheme and a two-tier scheme is location dependence. In a hierarchical scheme, the user identification numbers do not need to specify location information; therefore, the hierarchical scheme will also be referred to as a "location independent" scheme. The two-tier architecture is characterized as a "location dependent" database architecture. In this paper it is assumed that the wireless ATM network signaling uses a location dependent database architecture. We next consider the procedures used in the overlay approach to wireless ATM signaling.

\section{The Overlay APPROACH to WIRELESS ATM SignALING}

In this section, we discuss the network transactions that are related to supporting user mobility in the wireless ATM network and suggest how they can be implemented using the ATM User Network Interface (UNI) signaling protocol [6]. ${ }^{1}$ The network transactions related to user mobility are

1) registration;

2) call setup;

3) handoff.

We will refer to the operational software that manages a zone and the signaling systems in that zone as the "zone manager." The zone manager is responsible for database and connection management functions in the zone and runs on the wireless-to-ATM network interface equipment. There are two networks and two kinds of signaling in the overlay wireless ATM signaling network architecture. The zones are interconnected by an ATM network. The zone managers must

\footnotetext{
${ }^{1}$ ATM UNI (version 3.1) is an implementation agreement proposed by the ATM Forum and coincides with the ITU-Q.2931 signaling protocol implementation. In this text, the UNI signaling messages will be typeset in BOLDFACE characters. Note also that the zones of the wireless network will appear as "users" to the fixed ATM network; hence, use of ATM UNI signaling is justified.
}

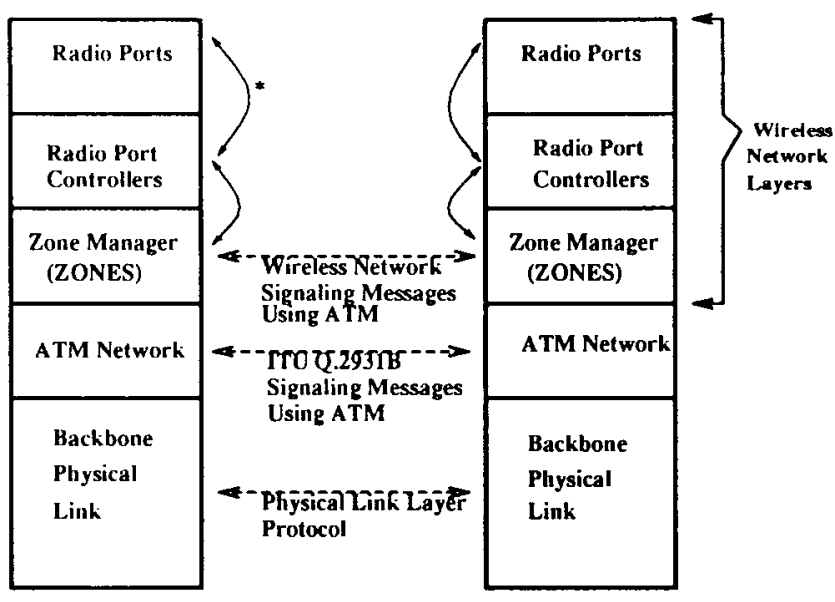

(*) The radio ports and port controllers can only communicate with the corresponding zone managers. The comm. between radio ports and port controllers is unspecified.

Fig. 2. Protocol layers in the wireless and ATM networks. The radio ports and the port controllers may only communicate with the zone managers. The links between the radio ports and the port controllers are not specified.

use the ATM signaling in order to establish connections through the ATM network. The radio ports and portables belong to the wireless network, and the zone managers exchange wireless network signaling messages in order to support the needs of the users and the network itself. The wireless network signaling messages are encapsulated into ATM cells and transmitted through the ATM network to their destinations. See Fig. 2 for a depiction of connection types and protocols between the layers in the wireless and ATM networks.

A procedure that is common to all of the wireless network transactions is establishing a connection through the fixed ATM network. The current ATM Forum UNI specification describes how an ATM connection is established in a fixed network context [6], using current ATM protocols between two ATM network entities. In this paper, registration, call setup and handoff will be implemented using the ATM connection setup procedure as described in [6] in an overlay manner. Note that this procedure is in a fixed network context and is used by all ATM network users. The calling/called parties will be zone managers in the wireless ATM network context. If the radio ports and the port controllers are also interconnected by the ATM network, then the same procedure could be used by the ports and controllers as well.

\section{A. Registration}

In the following sections, we show how the ATM connection setup procedure is used to implement registration, call setup, and handoff transactions for the wireless network.

Registration is performed to maintain information about the mobile user locations. The registration is performed as follows.

1) The registration process starts with the transmission of the user identification number (UID) and user's previous zone identification from the portable that enters a new zone. The portable recognizes the zone change by 
comparing the current zone identification with the stored last zone identification. The radio ports are assumed to transmit zone identification beacon signals periodically to assist the registration process [10], [11]. After exchange of encryption keys between the portable and the radio port, ${ }^{2}$ user's password is also transmitted over the radio interface in an encrypted message.

2) Upon receiving the UID and the authentication information, the zone manager ( $\mathrm{ZM})$ of the new zone establishes an ATM connection to the zone that contains the user profile ("home" zone). ${ }^{3}$ The location of the user profile may be obtained by querying the user's previous known zone. The zone managers communicate by using the wireless network signaling protocol messages that are encapsulated into ATM cells.

3) After the ATM connection between the new and home zone managers is established, the zone manager of the user's current zone requests user's authentication record and upon receipt of this record, the user is authenticated.

4) If authentication is successful, the user's profile is updated with the new location information and the updated profile is transferred to the current zone.

5) The user's profile in the previous zone is deleted by establishing an ATM connection to the previous zone manager.

6) If the authentication is unsuccessful then the user access is denied.

7) After the registration transaction is complete, the connection is released using RELEASE and RELEASE COMPLETE messages.

Please see Fig. 3 for message flows for a successful registration. ${ }^{4}$

\section{B. Call Setup}

The call setup procedure is used to establish a connection between two wireless network users. In this section, the originating zone refers to the calling user's zone and the destination zone refers to the called user's zone. The call setup proceeds as follows.

1) Called user identification number (CUID) is transmitted from the portable to the originating zone manager together with call setup parameters such as required bandwidth, traffic type, etc.

2) The originating zone manager (OZM) forms an ATM UNI protocol SETUP message using the incoming call parameters and proceeds as follows.

a) OZM requests the called user's location information from the wireless network. The procedure for this process is similar to what is described in Sections II and III-A and in Fig. 3.

\footnotetext{
${ }^{2}$ Note that if public key cryptography is being used the encryption public key maybe sent in the clear.

${ }^{3}$ Note that if the user profile is stored in a hierarchical database structure then a search has to be performed in order to locate the record; however, the steps involved in each step of the search will be the same as what will be described in the following paragraphs.

${ }^{4}$ The release of signaling connections are not shown for the sake of clarity of figures. They are included in our calculations.
}

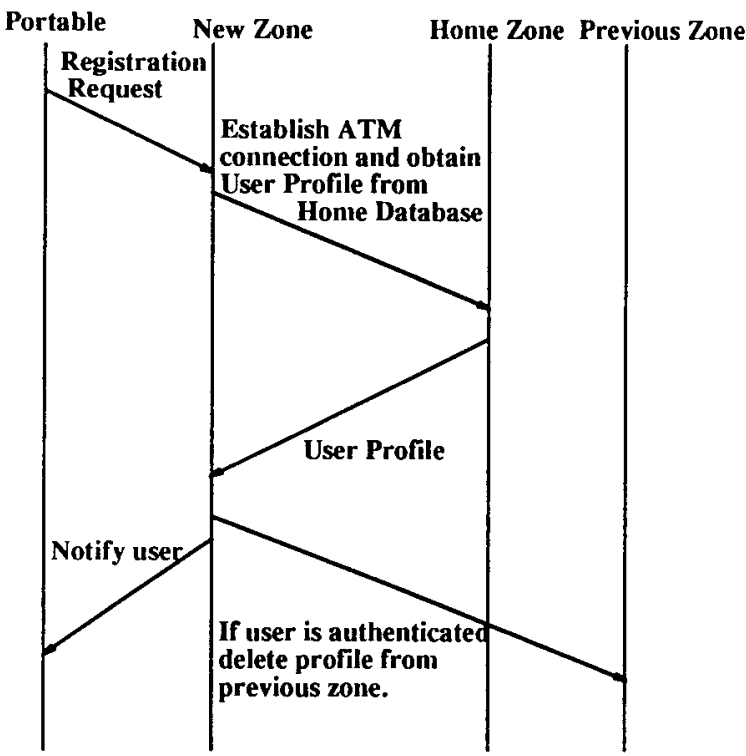

Fig. 3. Signaling message flow for registration; see footnote four.

b) When the called user's location information is retrieved from the network, an ATM connection is established to the called user's current zone manager using the ATM connection setup procedure.

c) After the connection is established, the OZM passes the calling user's identification number to the destination zone manager (DZM) and requests a connection setup. If the calling user's identification number is not in the call blocking list of the called user, then the called user is paged, if the page is successful, the DZM requests an ATM connection between the two users. If the page is not successful, then the called user is pronounced inactive and the proper record is updated. If the calling user is in the call blocking list of the called user then connection is rejected.

d) Pending ATM user connection establishment, the calling user and the called user are notified and the connection is established.

e) If the called user is busy, then the DZM notifies the OZM of the "busy" state and call attempt is terminated. The connection between the two zone managers is released.

3) After the call is complete, the ATM connection is released using RELEASE and RELEASE COMPLETE ATM signaling messages.

See Fig. 4 for the call setup transaction flow for a successful call.

Remarks:

1) Call blocking may be checked at the same time as the user's profile is obtained.

2) The connection setup for the user connection may be initiated by the OZM.

3) Since the connection involves radio links, a mechanism for call parameter renegotiation should be developed as the user environments vary or as the users change tiers in a multitier PCS environment [17]. 


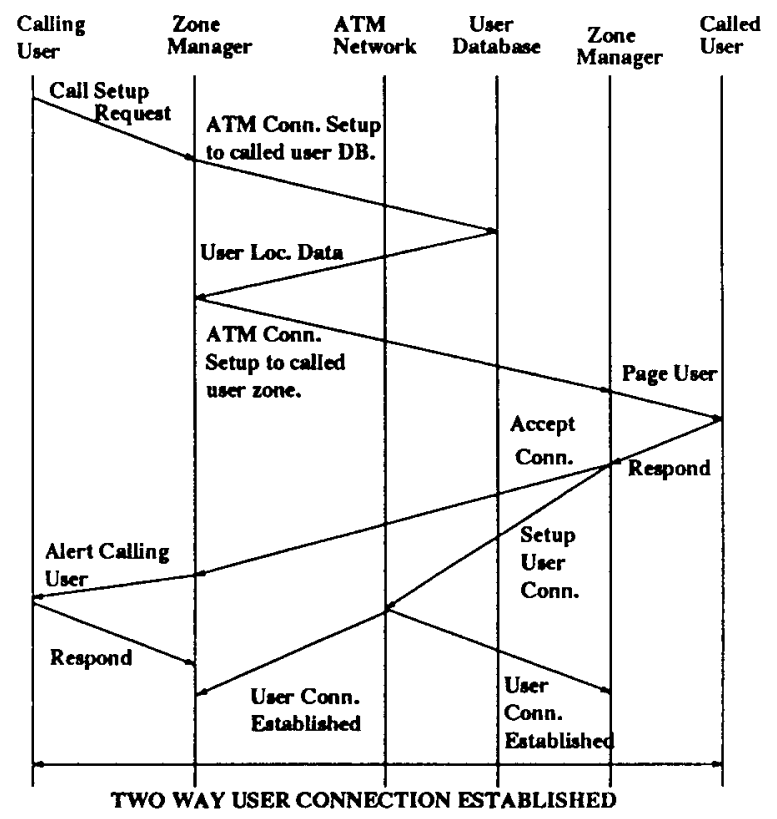

Fig. 4. Signaling message flow for call setup. The release of signaling connections is not shown in the figure to improve clarity but counted in the message statistics.

\section{Inter-Zone Handoff Using Overlay Signaling}

The inter-zone handoff process depends on its implementation in the wireless network. We will assume the following.

1) Handoff is portable initiated. The portables monitor the link quality in terms of received signal power to candidate radio ports and when the link to another port becomes stronger that port is selected and handoff is executed [11].

2) The handoff process may be initiated in two ways: The portable may tune into the control channel of the candidate radio port and initiate a handoff through the candidate port or the portable may use the existing link with the previous port to initiate the handoff. The latter method is used more frequently in present systems [11]; however, both methods have merit and will be discussed.

3) The only kind of handoff that generates network traffic is an inter-zone handoff. Therefore we limit the discussion to inter-zone handoffs. The intra-zone handoff is performed in a similar manner by the radio port controller without generating network signaling traffic.

Since we limit our discussion to the inter-zone handoffs, the candidate radio port and the previous radio port are in different zones. The candidate radio port is controlled by the "candidate zone manager." The previous radio port is controlled by the "previous zone manager." Please refer to Figs. 5-7 for details. The handoff will proceed as described below.

Case 1-Handoff Through the Previous Port:

1) In this case, the portable realizes that a link of better quality exists to a candidate radio port. The portable records the identity of the candidate port.

2) The portable sends a message to the previous zone manager (PZM) desiring a handoff to the candidate radio port. The ATM address of the candidate zone is included

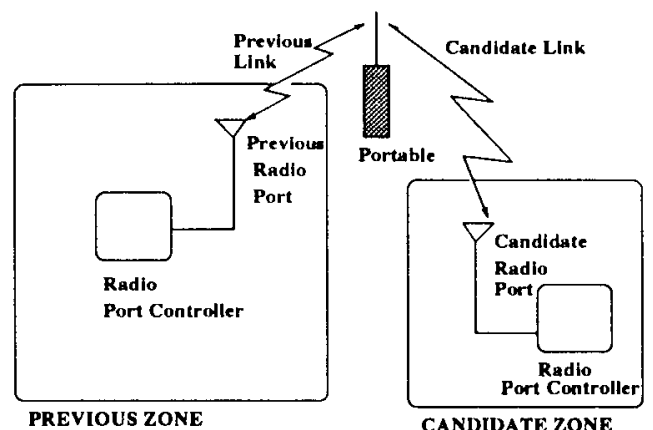

Fig. 5. The handoff: basic picture. Here the candidate zone isdesignated as a candidate for handoff. The previous zone is the zone the portable is communicating with before the handoff.

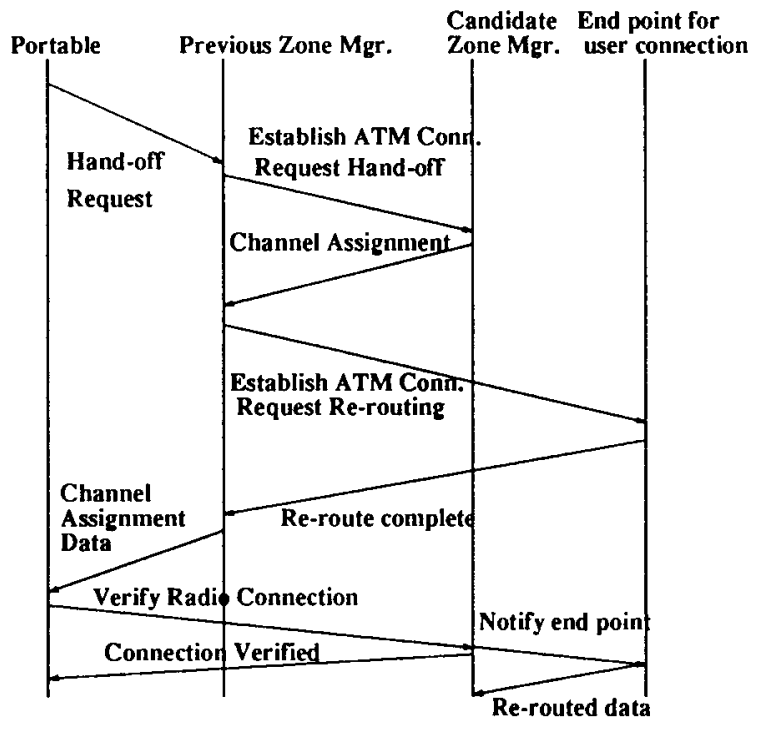

Fig. 6. Signaling message flow for successful handoff, Case 1, using previous port; see footnote four.

in this message. Since we are considering inter-zone handoffs the candidate radio port is in a different zone.

3) The PZM establishes an ATM connection to the candidate zone manager (CZM) using the ATM connection setup procedure.

4) The PZM transfers a copy of the user profile to the CZM, the CZM assigns a channel to the user and relays the channel assignment information to the PZM.

5) The PZM contacts the end point for the user connection and requests re-routing to the candidate zone. See [2] for details on rerouting of connections in a wireless ATM network.

6) Once the rerouting is complete, the PZM contacts the portable and relays the channel assignment information.

7) The portable tunes to the new channel and contacts the CZM.

8) The CZM and the portable verify the connection. After verification the CZM notifies the PZM of the successful handoff. If the connection is not verified, the portable tunes to the previous channel and starts scanning for candidate ports. The CZM deallocates the assigned channel. An alternative to this procedure is to verify the 


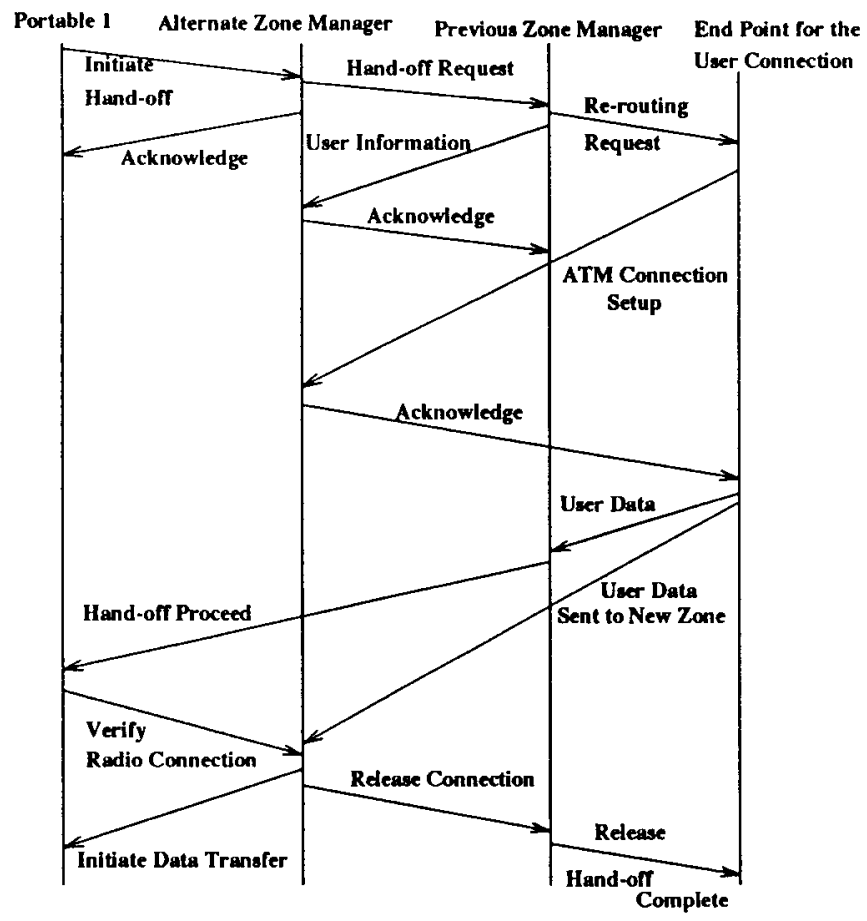

Fig. 7. Signaling message flow for successful handoff, Case 2, using candidate port; see footnote four.

radio channel and then perform the rerouting in order to save resources. However, since the handoff is portable initiated and depends on power measurements, success is more likely than failure.

9) If the handoff is successful, the PZM deletes user profile.

Case 2-Handoff Through the Candidate Port:

1) The portable initiates a handoff by establishing a link with the candidate radio port in the candidate zone and requesting a handoff from the candidate zone manager. This message includes the user's identification number and previous zone identification.

2) The candidate zone manager will acknowledge this message. The CZM will establish an ATM connection to the PZM using the ATM connection setup procedure. The portable will tune back to its previous channel after the acknowledgment is received.

3) Following the connection establishment, the CZM will request a handoff. The PZM will transfer the user profile to the CZM using the ATM connection. The PZM shall also send a re-routing message to the ATM network so that the route to the candidate zone can be setup. ${ }^{5}$ Rerouting of wireless ATM connections due to a handoff event is discussed in detail in [2].

4) The CZM shall provide the PZM with the channel assignment information for the portable. Pending rerouting success, the CZM will start to buffer the information for the portable. If the rerouting is not successful

\footnotetext{
${ }^{5}$ Currently, this can only be achieved using a SETUP message to establish the new connection. However, we can use the fact that the handoff is most likely to occur between neighboring zones and perform the re-routing at the nearest common network point, this capability would need to be added into the ATM network signaling protocol.
}

the handoff attempt is dropped, the PZM is notified. The PZM in turn notifies the portable.

5) If the rerouting is accomplished, the PZM sends the proceed with handoff message to the portable that is still tuned to the channel in the previous zone. The proceed with handoff message includes the channel assignment information for the candidate zone. If the portable cannot be raised at the previous zone, the CZM will be notified and the CZM will page the portable to establish a link. This page will also include the candidate channel assignment.

6) The portable, after receiving either the channel assignment information or the page from the CZM, will tune to the assigned channel and transmission is initiated. In either case the buffered information will be passed on to the portable in the order received. If the accepted time limits for the connection are exceeded then some information such as expired voice packets may be dropped,

7) When the handoff is completed, ${ }^{6}$ the ATM connection between the zone managers is released. Upon release of this connection the PZM will update the user's profile using a procedure similar to registration (authentication is not needed in this case) and erase the information that is buffered for the portable at the previous zone.

The differences between these two alternative methods are subtle; however, the former method (handoff through the previous port) does not need synchronization to the control channel of the candidate port and uses the existing radio link to the previous port for handoff related signaling. Note that if handoff is being performed because the existing link is deteriorating, then the success of the former method is not assured; nevertheless, if the portables monitor the link quality frequently then this will be a minor problem. The latter method suffers from difficulty of carrying on two connections at once but does not suffer from the failing link problem of the latter.

This completes the outline of the proposed implementation of a wireless ATM network that overlays the fixed ATM network. In the next section we describe the migratory signaling approach for wireless ATM network signaling.

\section{THE Migratory APPROACH TO WIRELESS ATM SigNALING}

Migratory signaling is a protocol implementation for a wireless ATM network that satisfies the goals listed below.

1) The signaling protocol shall support all the functions supported by the existing ATM signaling protocol and will require minimum change to the existing protocols.

2) The signaling protocol shall be migratory. It will support upgrading of the network in phases or in regions while maintaining compatibility with the existing network.

3) It shall be flexible and pave the way for future signaling network protocol implementations without major changes of the signaling network.

4) It shall address all the concerns listed for the overlay approach in Section III.

\footnotetext{
${ }^{6}$ The handoff is completed when the stability of the new link is established.
} 
The migratory signaling approach implements a single signaling protocol for support of both wireless and wireline users. We follow the procedure given in [1] and focus on how registration, handoff and connection setup are implemented in this new signaling protocol implementation. We refer to the new protocol as the "W-ATM signaling protocol" and use the words user and terminal to refer to a wireless ATM network endpoint. We also assume that a wireless ATM user's identification number uniquely determines the permanent home database for that user as described previously. The support for fixed ATM users is defined in ATM Forum and ITU documents [6], [7]. We therefore explain how the wireless users in the W-ATM network are supported and how the interaction between the fixed and wireless users is implemented. The fixed ATM users are supported by incorporating the current ATM protocol into the W-ATM Signaling Protocol.

\section{A. Registration Using the Migratory Signaling}

Registration in the W-ATM network is performed to keep the user location information current and is implemented as follows.

1) The user enters a new zone. The user terminal initiates a registration session with the zone manager for the new zone. After encryption key exchange (see footnote two), the terminal sends the user's identification number (IDN) to the zone manager and goes to standby state.

2) The zone manager records the IDN and sends a REGISTER message to the user's permanent registration database. ${ }^{7}$ The REGISTER message contains the user's identification number, the zone's ATM address, the user's permanent home database ATM address, and other standard ATM signaling parameters.

3) The REGISTER message is transmitted through the ATM network using the ATM signaling virtual circuits. The permanent home database receives the message and authenticates the user. Upon authentication it updates its records and sends two messages: REGISTER COMPLETE to the user's new zone and RECORD DELETE to the user's previous zone to delete user's record. ${ }^{8}$ The REGISTER COMPLETE message contains the user IDN, the ATM address of the current zone, the user profile, and the ATM address of the home database of the user. The RECORD DELETE message contains the user IDN, the ATM address of the previous zone, and the home database of the user.

4) If the authentication is not successful, then the user's new zone is notified by sending a REGISTER DENY message.

5) If the registration is successful, the user's terminal is notified; for unsuccessful registration attempts the registration session is re-initiated. If the registration is unsuccessful a second time then the user is denied service and the network operations control is notified.

\footnotetext{
${ }^{7}$ Throughout this section, we will use BOLDFACE letters to differentiate new W-ATM signaling messages. See [5] for details on the contents of W-ATM signaling messages.

${ }^{8}$ RECORD DELETE message is not sent in the so-called lazy deregistration schemes.
}

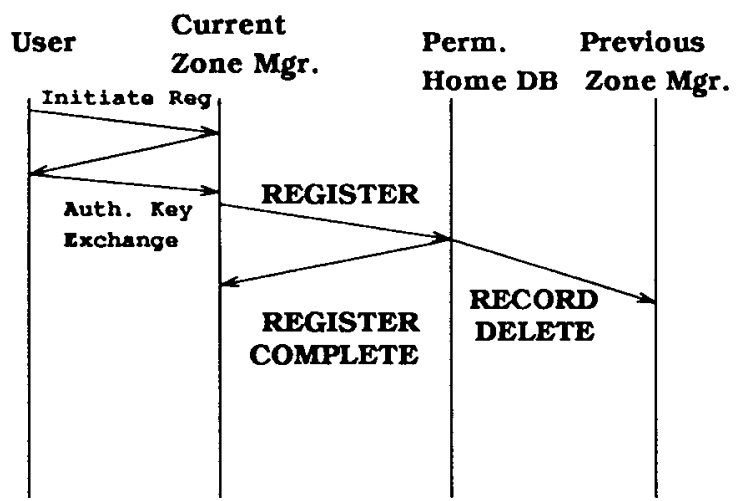

Fig. 8. Signaling message flow for successful registration.

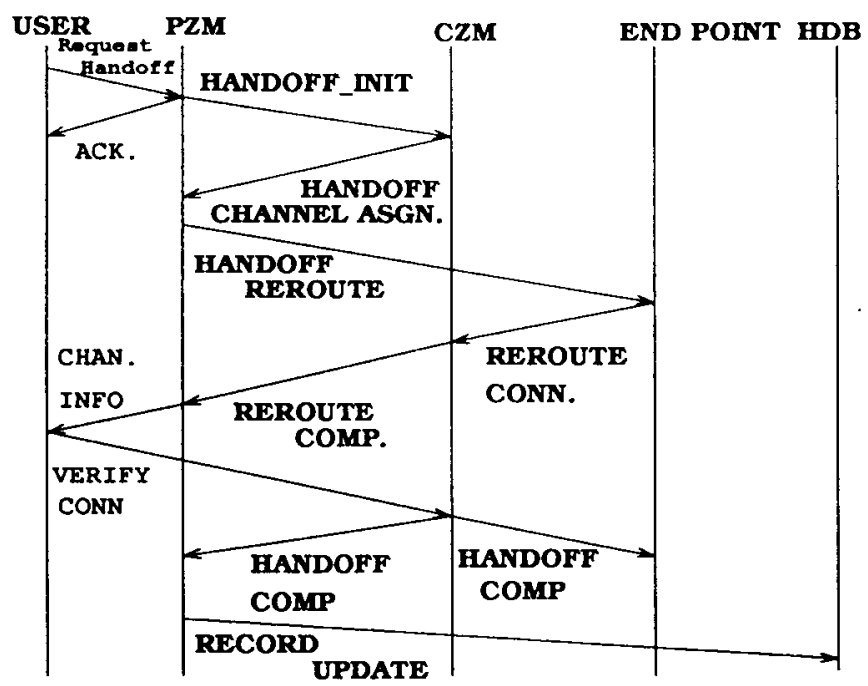

Fig. 9. Signaling message flow for successful handoff, Case 1, through the previous port.

The signaling message flow for registration is given in Fig. 8 . Note that there are no overlay ATM connections established for this process. All signaling messages are W-ATM native and use ATM signaling circuits to traverse the network.

\section{B. Inter-Zone Handoff Using the Migratory Signaling}

As in the discussion of handoff given in Section III-C, we concentrate on the inter-zone handoff; therefore, the candidate radio port and the previous radio port are in different zones. We assume that the handoff is portable initiated and assisted and that the handoff may either be performed through the candidate or through the previous radio port. See [1], [18], and [22] for details on handoff. The candidate radio port is controlled by the CZM. The previous radio port is controlled by the PZM. Please refer to Figs. 5, 9, and 10 for details. The handoff will proceed as described below.

\section{Case 1-Handoff Through the Previous Port:}

1) In this case, the portable realizes that a link of better quality exists to a candidate radio port. The portable records the identity of the candidate port.

2) The portable sends a message to the PZM requesting a handoff to the candidate radio port. Since we are 


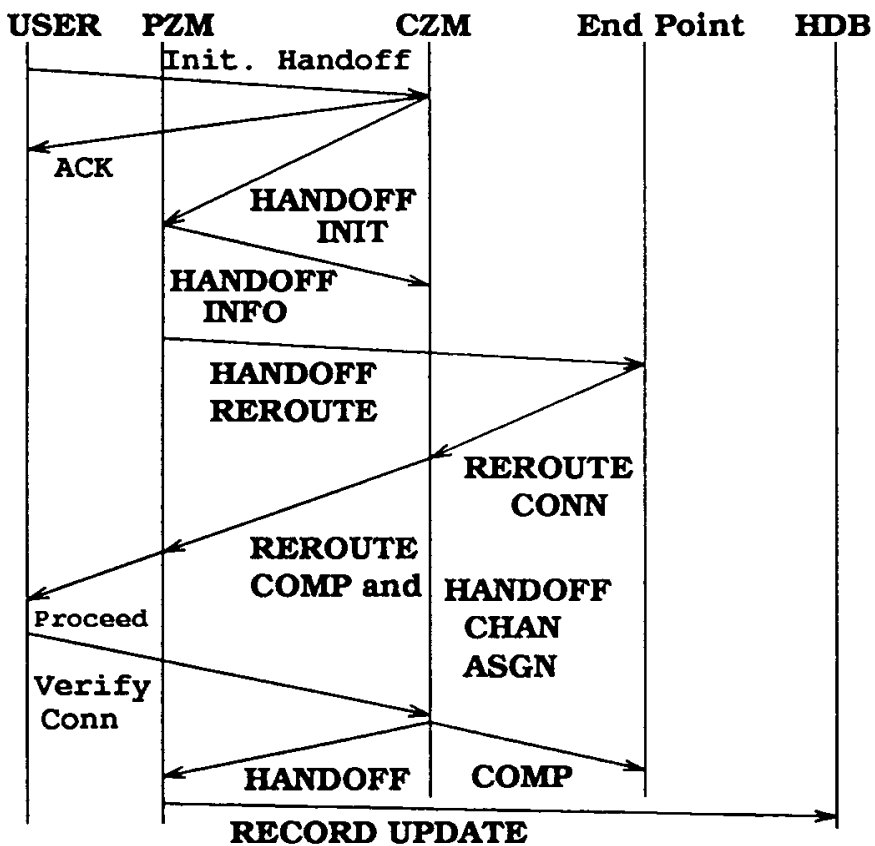

Fig. 10. Signaling message flow for successful handoff, Case 2, using candidate port.

considering inter-zone handoffs the candidate radio port is in a different zone managed by the CZM.

3) The PZM sends a HANDOFF INIT message to the CZM. This message contains the ATM addresses for the PZM and CZM and the user's IDN and profile.

4) CZM receives the HANDOFF INIT message and assigns a channel to the user relays the channel assignment information to the PZM by sending a HANDOFF CHANNEL ASSIGN message to the PZM.

5) The PZM notifies the end point ${ }^{9}$ for the user connection by sending a HANDOFF REROUTE message. The HANDOFF REROUTE message contains the address of the candidate zone and the user's IDN.

6) The end point for the user connection sends a REROUTE CONNECTION message to the CZM. The end point at this stage also stops sending information to the PZM and starts buffering the information. The reroute is performed at the point where the route to the PZM and the route to the CZM forks. The CZM sends a REROUTE COMPLETE message to the PZM. See [2] for detailed description of rerouting due to a handoff in wireless ATM Networks.

7) Once the rerouting is complete, the PZM contacts the portable and relays the channel assignment information.

8) The portable tunes to the new channel and contacts the CZM.

9) The CZM and the portable verify the connection. After verification the CZM notifies the PZM of the successful handoff by sending a HANDOFF COMPLETE message. Upon receiving the HANDOFF COMPLETE, PZM releases the connection between itself and the

\footnotetext{
${ }^{9}$ The end point is defined to be the terminating node for the user connection in the network.
}

end point [2]. The CZM sends a HANDOFF COMPLETE to the end point to resume data transfer. If the connection is not verified, the portable tunes to the previous channel and starts scanning for candidate ports. The CZM deallocates the assigned channel and sends a HANDOFF FAIL message to the PZM and the end point. The end point starts sending the data to the PZM. Note that some time-sensitive information such as voice may be discarded if the handoff takes longer than prespecified time interval.

10) When the handoff is completed, ${ }^{10}$ the PZM deletes the user profile and sends a RECORD UPDATE message to update the user profile to the user's permanent home database. This message contains the user's IDN and the ATM address for the candidate zone.

Case 2-Handoff Through the Candidate Port:

1) The portable initiates a handoff by establishing a link with the candidate radio port in the candidate zone and requesting a handoff from the candidate zone manager. This message includes the user's identification number and previous zone identification.

2) The CZM will acknowledge this message. The CZM will send a HANDOFF INIT W-ATM message to the PZM. The HANDOFF INIT message contains the user's IDN and the address for the PZM and the CZM. The portable will tune back to its previous channel after the acknowledgment is received.

3) Upon receiving the HANDOFF INIT message the PZM will transfer the user profile to the CZM using a HANDOFF INFO message. The PZM shall also send a HANDOFF REROUTE message to the end point for the user's connection. The rerouting is performed as specified in Section IV-B1 and as described in [2].

4) If the rerouting is successful, the CZM shall provide the PZM with the channel assignment information for the portable using a HANDOFF CHANNEL ASSIGN message. It will also send a REROUTE COMPLETE message to the end point. If the rerouting is not successful, the handoff attempt is dropped, the PZM and the end point are notified by sending a HANDOFF FAIL message listing rerouting failure as the reason. The PZM in turn notifies the portable.

5) If the rerouting is accomplished, the PZM sends the proceed with handoff message to the portable that is still tuned to the channel in the previous zone. The proceed with handoff message includes the channel assignment information for the candidate zone. If the portable cannot be reached at the previous zone, the CZM will be notified and the CZM will page the portable to establish a link. This page will also include the candidate channel assignment.

6) The portable, after receiving either the channel assignment information or the page from the CZM, will tune to the assigned channel and transmission is initiated. The CZM will send a HANDOFF COMPLETE message to the the PZM and the end point for the connection.

\footnotetext{
${ }^{10}$ The handoff is completed when the stability of the new link is established
} 


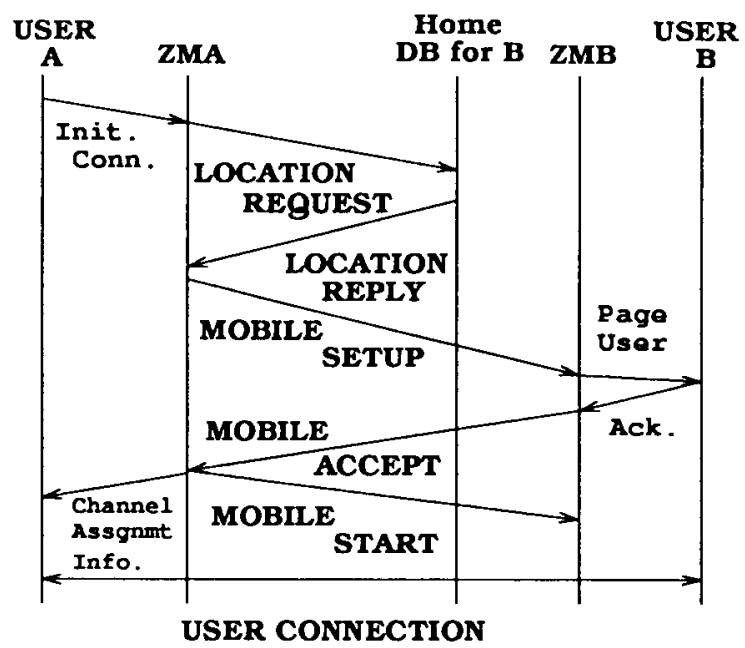

Fig. 11. Successful connection setup between two wireless users in different zones.

The end point then will release the data that is being buffered. At this stage the PZM releases the connection between itself and the end point [2].

7) When the handoff is completed (see footnote 10), the PZM deletes the user profile and sends a RECORD UPDATE message to update the user profile to the user's permanent home database. This message contains the user's IDN and the ATM address for the candidate zone.

\section{Connection Setup Using Migratory Signaling}

The connection setup procedure in the W-ATM network must be investigated in three stages, as follows:

1) connections from wireless to wireless users;

2) connections from wireless to fixed users;

3) connections from fixed to wireless users.

The connection setup from fixed users to fixed users is handled adequately by the current ATM signaling specifications hence is not discussed here [6], [7].

We assume in this paper that the wireless users are mobile and change their locations. The user's locations are updated by means of the registration process described in the previous sections. The main differentiator between connections in the ATM network and the connections in the wireless ATM network is the address or location resolution process performed before an actual connection is established; therefore, the standard ATM connection setup procedure becomes inadequate. We propose the following W-ATM connection setup procedure for setting up connections in the W-ATM network. We consider the three cases mentioned previously:

Case 1-Connection Setup Between Two Wireless Users: We assume that the wireless users and the fixed users are differentiated by their user identification numbers. The ATM address for a fixed ATM terminal is recognized as the IDN. For this case we will assume that wireless user $A$ decides to call wireless user $B$. The procedure for connection setup is given below and illustrated in Fig. 11.

1) User $A$ 's terminal contacts the zone manager for $A$ 's current zone. The terminal transfers the IDN for user
$B$ and the connection setup parameters to the zone manager. The zone manager for user $A$ is referred to as the "ZMA" throughout the section.

2) User $B$ 's IDN indicates that $B$ is a wireless user. The ZMA checks the visitor location register to see if $B$ is in the same zone.

3) If $B$ is in the same zone as $A$ then the ZMA allocates channels for both users and sends the assignment information to both $A$ and $B$ over radio links. This completes the connection setup process.

4) If $B$ is not in the same zone as $A$ then the ZMA starts a connection setup session. A connection setup session consists of two stages: the location resolution and the connection establishment. In the location resolution stage the ZMA finds the current location of user $B$. In the connection establishment stage the ZMA establishes the actual user connection between $A$ and $B$.

5) Location Resolution: The ZMA uses $B$ 's IDN to resolve the ATM address of the permanent home database of $B$. The ZMA sends a LOCATION REQUEST message to $B$ 's permanent home database. The LOCATION REQUEST message contains user $B$ 's IDN, the ATM addresses of the ZMA and $B$ 's permanent home database. The permanent home database of user $B$ replies by sending a LOCATION REPLY message to the ZMA. The LOCATION REPLY is sent if and only if the calling user $(A)$ is not in called user's $(B)$ call blocking list. If user $A$ is in the call blocking list, the permanent home database will send a REQUEST DENIED message to the ZMA stating the call blocking as the reason. The LOCATION REPLY message contains $B$ 's present location and the ATM address for the ZMA. The call blocking is checked at the home database for security reasons.

6) Connection Establishment: After retrieving the location information for user $B$, the ZMA contacts the zone manager of $B$ 's present zone (ZMB) by sending a MOBILE SETUP message. The MOBILE SETUP message contains the ATM address of ZMB, the user IDN for $B$ and the connection parameters for the user connection. The intermediary nodes that receive this message reserve the appropriate resources and set up the virtual circuit translation tables for the connection. If an intermediary node is not able to allocate the required resources, it sends a MOBILE SETUP DENIED message to the ZMA, clearing the established circuits in the intermediary nodes on the path. If the MOBILE SETUP message reaches the ZMB, this means that the path throughout the network for the user connection is established and the resources are allocated. The ZMB pages user $B$.

a) If $B$ responds to the page, then the $\mathrm{ZMB}$ allocates the radio channel for the user connection and relays this information to $B$. The ZMB then sends a MOBILE ACCEPT message to the ZMA and confirms the connection.

b) If $B$ does not respond to the page then $B$ is assumed to be in inactive stage and the ZMB sends MOBILE 
SETUP DENIED message to the ZMA, releasing the connection in the network.

7) If the user connection request is accepted by the $\mathrm{ZMB}$, then the ZMA pages $A$ and relays channel assignment information. The ZMA sends a MOBILE START message to the ZMB indicating the start of user data. User $A$ starts sending information.

8) If the user request is denied, the ZMA relays this information to $A$.

Case 2-Connection Setup from Wireless to Fixed Users: For this case, we assume that wireless user $A$ decides to establish a connection to fixed ATM terminal $B$. $B$ 's ATM address is used as the user IDN for connection purposes. A connection from $A$ to $B$ is established as follows and is illustrated in Fig. 12.

1) User $A$ establishes a radio link to the zone manager for $A$ 's current zone (ZMA). It requests a connection to be set up between $A$ and $B$. The ZMA recognizes $B$ 's IDN as being a fixed ATM terminal.

2) Since the called user, $B$, is a fixed ATM terminal, there is no need for location resolution for this case. The ZMA constructs a MOBILE SETUP message to $B$. This mobile setup message contains: $A$ 's IDN, the ZMA's ATM address, $B$ 's ATM address and the connection setup parameters.

3) The MOBILE SETUP message sent by the ZMA is sent through ATM signaling virtual circuits. Each intermediary node that receives this message tries to allocate the resources required by the connection and sets up the translation tables. If the required resources could not be allocated for the connection, the intermediary node sends a MOBILE SETUP DENIED message to the ZMA clearing the connection at all the nodes on the path from itself to the ZMA. The MOBILE SETUP DENIED message cites insufficient resources as the reason for denying the request.

4) If all the intermediary nodes between the ZMA and the fixed ATM host $B$ are able to allocate the desired resources, the MOBILE SETUP message is received by $B$. $B$ compares $A$ 's ID against its call blocking list. If $A$ is in the call blocking list, $B$ sends a MOBILE SETUP DENIED message. If $A$ is not in the call blocking list, $B$ sends a MOBILE ACCEPT message to $A$. This message is received by all of the intermediary nodes and virtual circuits are set up.

5) When the MOBILE ACCEPT message is received by the ZMA, wireless user $A$ is paged. After $A$ responds to the page, the ZMA sends a MOBILE START message to $B$. The connection is established. If $A$ does not respond to the page, then the connection is cleared.

Case 3-Connection Setup from Fixed to Wireless Users: In this case we assume that a fixed ATM terminal $A$ requires a connection to be established to a wireless user $B$. The ATM address for $B$ is equivalent to $B$ 's IDN. The connection setup proceeds as follows and is illustrated in Fig. 13.

1) The fixed ATM user $A$ determines that $B$ is a wireless terminal by the given ATM address for $B$. It then decides

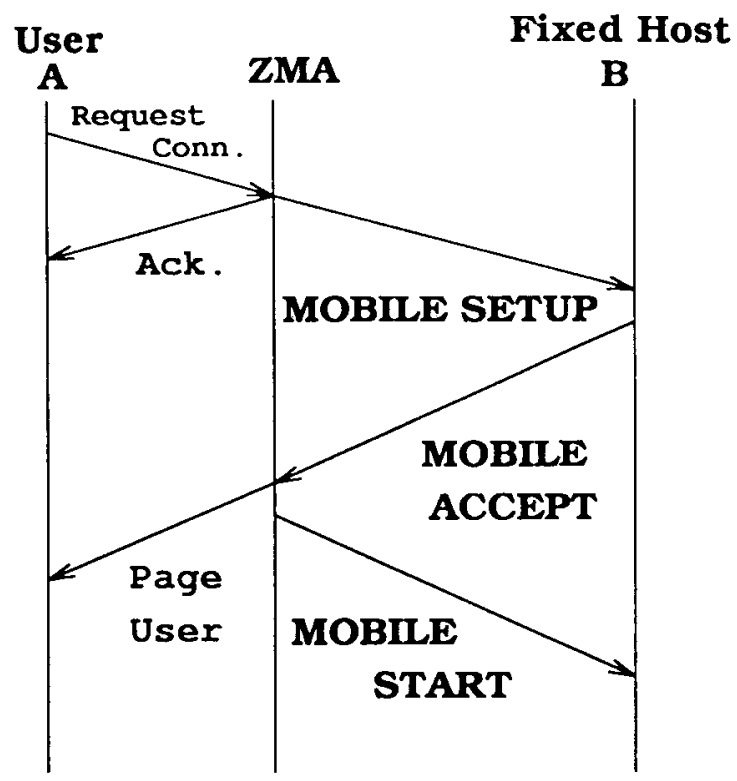

Fig. 12. Successful connection setup from a wireless to a fixed user.

to employ the W-ATM connection setup procedure to establish the connection. This proceeds in two stages.

2) Location Resolution: The fixed ATM user $A$ resolves the ATM address of the permanent home database for $B$. $A$ then sends a LOCATION REQUEST message to $B$ 's permanent home database. This message contains $B$ 's IDN, the ATM address of $A$ and $B$ 's permanent home database. The permanent home database of $B$ replies by sending a LOCATION REPLY message to $A$. The LOCATION REPLY message is sent if and only if $A$ is not in $B$ 's call blocking list. Otherwise, a REQUEST DENIED message is sent as a reply stating call blocking as a reason. The LOCATION REPLY message contains $A$ 's ATM address, $B$ 's IDN, and the ATM address of $B$ 's current zone.

3) Connection Establishment: After receiving the LOCATION REPLY message from the permanent home database of user $B, A$ sends a MOBILE SETUP message to the current zone of user $B$. This message contains $A$ 's ATM address, $B$ 's IDN, the ATM address of the current zone of $B$, and connection parameters. We will refer to the zone manager of current zone of user $B$ as the ZMB. All the intermediary nodes that receive the MOBILE SETUP message are required to allocate the necessary resources for the connection and setup the virtual circuit translation tables. If an intermediary node does not have the necessary resources, then it sends a MOBILE SETUP DENIED message to $A$ clearing the connection. The MOBILE SETUP DENIED message cites insufficient resources as the reason for denying the request. If the resources for the connection are available and the network path for the connection is established at the intermediary nodes, then the MOBILE SETUP message reaches the ZMB. Upon receiving the MOBILE SETUP message, the ZMB pages user $B$. 


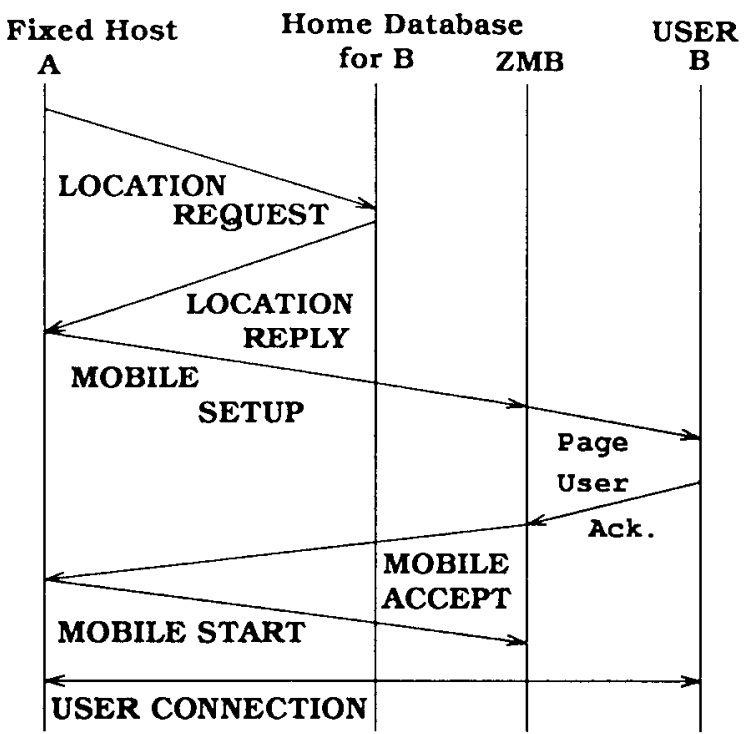

Fig. 13. Successful connection setup from a fixed to wireless user.

a) If $B$ responds to the page, then the $\mathrm{ZMB}$ allocates the radio channel for the user connection and relays this information to $B$. The ZMB then sends a MOBILE ACCEPT message to the fixed ATM user $A$ and confirms the connection.

b) If $B$ does not respond to the page then $B$ is assumed to be in inactive stage and the ZMB sends MOBILE SETUP DENIED message to the $A$ thereby releasing the connection in the network.

4) If the user connection is accepted by the $\mathrm{ZMB}, A$ sends a MOBILE START message to the ZMB and the connection is established.

Clearing an Existing User Connection: Clearing an existing user connection is performed in the same manner for all three cases listed above. The side that decides to terminate the connection sends a MOBILE CLEAR message to the other end point. The end point upon receiving the message sends a MOBILE CLEAR ACK message to the side that initiates the call clearing. All the intermediary nodes that receive the MOBILE CLEAR ACK message clear the connection. The end point sends the MOBILE CLEAR ACK message after all the outstanding data cells are received. By clearing the connection with the acknowledgment message the network ensures that there are no cells lost because of premature termination.

\section{ESTIMATED SignALING OVERHEAD REQUIRED TO SUPPORT WIRELESS USERS}

Estimating the performance of a signaling network protocol requires the definition of a performance measure. Our performance measure is the overhead required for signaling. We are not concerned with the signaling information being exchanged, but in the overhead required to carry that information. This performance measure is chosen because the signaling information needed to be communicated to perform network functions in any network remains roughly the same; however, the work performed in transmitting that information is a good measure of the efficiency of that protocol.

We used the methods given in [3] and [15] to estimate the signaling overhead required to support wireless users. These methods use the uniform fluid flow model for approximating wireless user motion, and Poisson call arrival and exponential call holding time distributions given in [12]. Equations that yield mean values for the registration, $L_{r}$, connection setup, $L_{c}$, and handoff, $L_{h}$, transaction rates in terms of transactions per second (TPS) are given in [15]. We have adapted and further simplified the equations given in [15] and arrived at the following set of equations (see Appendix B):

$$
\begin{aligned}
L_{c} & =N p c(1+q) \\
L_{r} & =\frac{(1-s)(1-m)}{\Pi} N \frac{4}{T} \\
L_{h} & =\frac{(1-s)(1-m)}{\Pi} N e \frac{4}{T} \\
T & =\frac{D}{v} .
\end{aligned}
$$

See Table III for the definitions of the terms. The equations above yield results for any given configuration that are accurate estimates within the limitation of being mean values for statistical variables. ${ }^{11}$ By examining the equations given above, the following are evident.

1) All of the transaction rates scale linearly with the number of people in a zone.

2) Assuming that the population density remains constant, all of the above transaction rates vary linearly with the square of the zone size.

3) The rate of registration transactions is not affected by call arrival rate or call holding time.

4) The rate of connection attempts is not affected by the user velocities.

5) Both the registration and handoff transaction rates vary linearly with user velocities assuming that zone size and thus the number of people in the zone remain the same.

We now proceed to discuss the performance estimates for both overlay and migratory signaling approaches. We note that the focus of the results are toward the effects of user velocity, calling rate and call holding time. Although we have performed all of the variations listed above, we only show a few examples of the linear relationships.

\section{A. Performance of the Overlay Signaling Approach}

In this section, we estimate the performance of the overlay signaling approach. For the estimates, we also specify the kind of ATM signaling needed to handle registration, handoff and connection setup transactions. The number of ATM connections that are required to be established for overlay signaling, as noted in Section III, are summarized in Table I. Based on these figures and the minimum and maximum number of bytes required to set up ATM connections (see Appendix A and [6]), we then calculate the mean number of transactions per

\footnotetext{
${ }^{11}$ The validity of these equations is verified in [15] and by independent simulations performed by the authors.
} 
TABLE I

Wireless ATM Signaling Traffic in Terms of ATM Connections That Need To Be Established

\begin{tabular}{l|r}
\hline Signaling Transaction & Number of ATM Connections \\
\hline \hline Registration (Explicit Deregistration) & 2 \\
\hline Registration (Implicit Deregistration-Lazy) & 1 \\
\hline \hline Connection Setup (Mobile to Mobile) & 3 \\
\hline Connection Setup (Mobile to Fixed) & 1 \\
\hline Connection Setup (Fixed to Mobile) & 3 \\
\hline Handoff & 4 \\
\hline \hline
\end{tabular}

TABLE II

Varied Parameters (Zone Size is 8000 m Unless Otherwise Stated)

\begin{tabular}{l|r}
\hline Varied Parameter & Values \\
\hline \hline $\begin{array}{l}\text { Call Arrival Rate } \\
\text { (Call Rate) }\end{array}$ & $1-6$ calls $/$ hour \\
\hline Call Holding Time & $60-360$ seconds \\
\hline Velocity of users (Zone Size $=8000 \mathrm{~m}$.) & $10-90 \mathrm{kmph}$ \\
\hline $\begin{array}{l}\text { Velocity of Users (Zone Size }=16000 \mathrm{~m} .) \\
\text { Zone Size }\end{array}$ & $10-90 \mathrm{kmph}$ \\
\hline
\end{tabular}

second and the mean bandwidth required for setting up ATM connections to support these transactions.

The parameters in Table II were varied one at a time to arrive at the results discussed in the text. Figs. 14 and 15 show examples of the linear relationships.

1) The ATM overlay signaling bandwidth required to support the wireless network is on the order of a few Mbits per second for all the cases investigated. We conclude that the bandwidth required to support the zones of the wireless network is minimal.

2) The number of ATM connections that need to be established for supporting the wireless network mobility; however, is on the order of 100 to 400 connection attempts per second. We note that this figure does not include the user connections. This figure is a very significant overhead and translates to roughly one connection setup every $2.5 \mathrm{~ms}$. To the best of the authors' knowledge this appears to be a challenge to current ATM switching technology.

3) All the figures given are for a square zone size of 8000 meters on one side. As the zone size increases, all of the quantities listed above either increase quadratically or linearly with the zone size. Thus, the overlay signaling paradigm does not scale well for supporting a large number of mobile wireless users.

4) The call holding time has little effect on the estimated ATM bandwidth required for signaling. Of course, it has a very marked effect on the bandwidth required to support user connections.

\section{B. Performance of the Migratory Signaling Approach}

In this section, we focus on the performance of the migratory signaling discussed in Section IV. We again use the mean number of signaling messages per second and the bandwidth required to transmit these messages as a performance criteria for migratory signaling. After performing the parameter variations listed in Table II we have observed the following.
TABLE III

Default Values Used in the Calculations

\begin{tabular}{l|r|r}
\hline Parameter Name & Parameter Symbol & Default Value \\
\hline \hline $\begin{array}{l}\text { Number of Users } \\
\text { in a Zone }\end{array}$ & $\mathrm{N}$ & None \\
\hline $\begin{array}{l}\text { Average Zone } \\
\text { Crossing Time }\end{array}$ & $\mathrm{T}$ & None \\
\hline $\begin{array}{l}\text { Call Arrival Rate } \\
\text { (Call Rate) }\end{array}$ & $\mathrm{c}$ & 3 calls/hour \\
\hline Call Holding Time & $\mu$ & 180 seconds \\
\hline Velocity of users & $v$ & $35 \mathrm{kmph}$ \\
\hline $\begin{array}{l}\text { Zone Size } \\
\text { (one side of the square) }\end{array}$ & $D$ & 8000 meters \\
\hline Penetration Ratio & $p$ & 0.25 \\
\hline $\begin{array}{l}\text { Successful Call } \\
\text { Completion }\end{array}$ & $q$ & 0.5 \\
\hline $\begin{array}{l}\text { Stationary User } \\
\text { Percentage }\end{array}$ & $s$ & 0.50 \\
\hline $\begin{array}{l}\text { Ratio of Mobile Users } \\
\text { that stay in one zone }\end{array}$ & $m$ & 0.45 \\
\hline $\begin{array}{l}\text { Probability of a } \\
\text { user being busy }\end{array}$ & $e$ & $\mu \times c$ \\
\hline
\end{tabular}

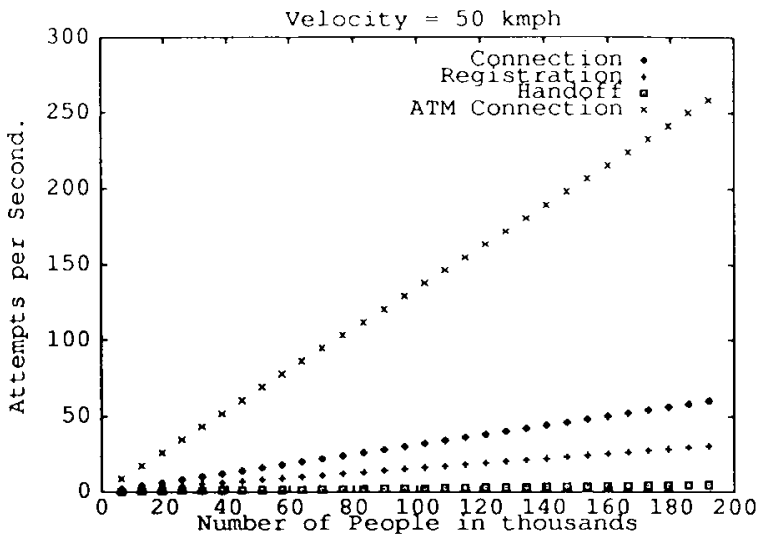

Fig. 14. Overlay signaling transaction rates. Zone size $=8000 \mathrm{~m}$, velocity $=50 \mathrm{kmph}$.

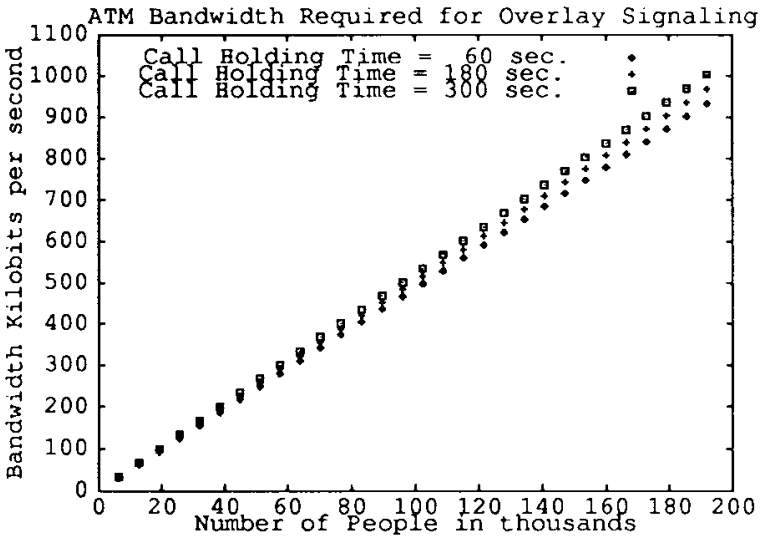

Fig. 15. Overlay signaling bandwidth versus call holding time.

1) The number of signaling messages sent and the bandwidth required to send them varies linearly with velocity, population of a zone and calling rate. Mean call holding time does not have a significant effect on the bandwidth or the number of signaling messages transmitted through the network. 


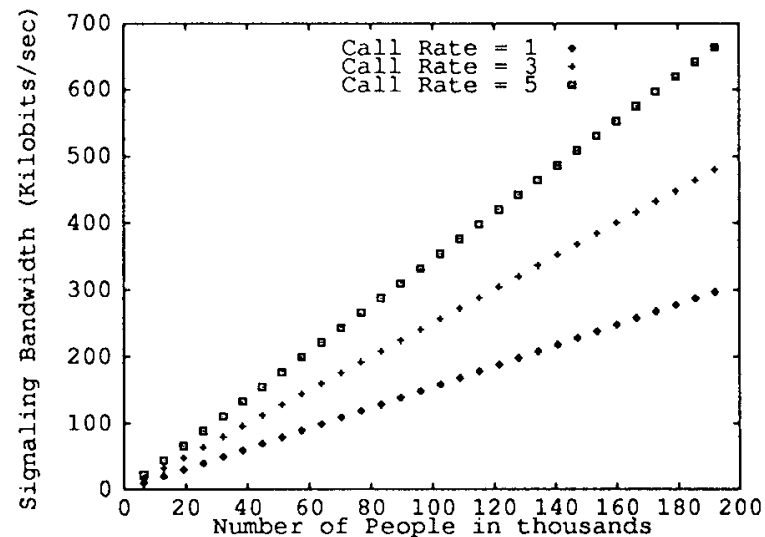

Fig. 16. Variation of required bandwidth by call arrival rate in migratory signaling.

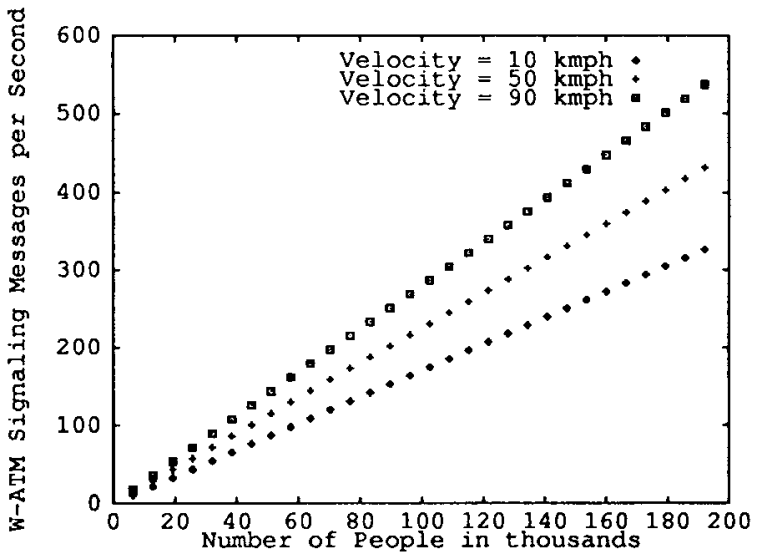

Fig. 17. Variation of the number of messages by velocity.

2) The mean number of signaling messages transmitted per second in the W-ATM network varies between 20 and 400 messages per second depending on the population and other factors.

3) The mean signaling bandwidth required to support WATM signaling varies between 10 and 900 kilobits per second depending on the population size and other factors. These bandwidth figures were calculated using the proposed W-ATM signaling messages defined in [5].

4) The single most dominant factor for all measures of interest is the population of the zone. For a given population both mean calling rate and mean user velocity are equally dominant. The call holding time has no significant effect for signaling although its effect on user bandwidth is very important.

Figs. 16 and 17 show examples of the linear relationships. Based on these calculations we can now compare the overlay wireless ATM signaling with migratory wireless ATM signaling.

\section{Comparison of the Overlay and Migratory Signaling Methods}

In this section, we compare the migratory wireless ATM signaling and the overlay ATM signaling. The overlay signaling is designed to support wireless users in an ATM network with no modification to the existing ATM protocols and only the addition of gateways to the ATM network. The migratory signaling is designed to be a native wireless ATM protocol providing built-in support for both wireless and wireline users. To achieve built-in support, migratory signaling adds a few signaling messages to the existing ATM protocols. The comparisons follow below.

1) The overlay signaling requires ATM connections to be established to support mobile (wireless) users. In Section III, we have shown that for varying population sizes, overlay signaling requires 40 to 250 ATM connections per second to be established resulting in the transmission of 200 to 1250 signaling messages per second. This presents a challenge to existing ATM switching equipment. The migratory signaling uses ATM signaling virtual circuits to transmit signaling message to support wireless users. Since these messages are sent using the existing ATM signaling virtual circuits, no connections need to be established. The number of mean signaling messages sent are on the order of 20 to 400 messages per second. This number is feasible using today's technology.

2) The bandwidth required to support signaling for wireless users is also an important criteria. Using our calculations we have found that the overlay signaling requires an average signaling bandwidth of $1.4 \mathrm{Mb} / \mathrm{s}$. For the same set of baseline parameters the migratory signaling requires approximately $500 \mathrm{~Kb} / \mathrm{s}$ of average bandwidth.

3) The interaction between wireless and wireline users in the wireless ATM network is an important feature of our design. In the overlay signaling, this interaction is implemented by means of service gateways that function as intermediary points between the wireline and wireless users. In the migratory signaling, the support for wireless and wireline user interaction is built-in to the signaling protocol. This native support is also more efficient in terms of bandwidth and signaling routing since the connections need not be routed through the service gateways.

4) We have mentioned in Section III that the overlay approach may have a scaling problem when the population of a zone exceeds 100000 people. The solution for this problem is further subdivision of zones with large population sizes. The migratory signaling is more robust in terms of scalability and it minimizes the scalability problem by using native signaling channels.

5) The overlay signaling requires no modification to the existing ATM protocols. The only ATM network modification required for overlay signaling is the establishment of service gateways in the networks as discussed previously. The migratory signaling requires addition of some signaling messages to the existing protocol to provide direct support for wireless users.

6) The inefficiency associated with overlay signaling is a result of choosing to use switched virtual circuits (SVC's) for signaling connections. An alternative to the use of SVC's is to use permanent virtual circuits (PVC's) for overlay signaling. By using PVC's, the 
zone managers of the wireless ATM network will be able to communicate without establishing connections. In such a network, the overhead associated with using PVC based overlay signaling will be comparable to migratory signaling discussed in this paper. However, there are some disadvantages associated with PVC based signaling as noted previously and in [4]. The primary disadvantage is the need for manual administration of PVC's for each and every zone; moreover, the number of required PVC's will grow with the square of the number of zones in the network. Due to space limitations, we cannot consider the use of PVC's herein. However, we note that architectures given in [4], [19] address the problems associated with PVC based overlay signaling. Also, most of the PVC based schemes require changes in the current ATM protocols. In contrast, SVC based overlay signaling does not require any changes in the existing protocols, it is completely distributed and requires no administration.

We also note here that these protocols may be used in tandem to evolve support for wireless users in an ATM network to form a "wireless" ATM network.

\section{IMPACT OF MOBILITY ON THE ATM NETWORK}

In this section, we discuss the impact of mobility on the current ATM networks that are being designed to handle fixed users. In these fixed networks, the user's identification number determines where the user is and how to route a call to the user. However, in a wireless environment, the user numbers do not yield any information about the user's location. In such an environment the user's number must be used to retrieve the location information. Note that if the wireless and ATM networks were separate entities in which no cross-interaction between users occurred, then the discussions in Section III would provide a way of implementing a selfcontained wireless network supported by an ATM network. However, the users in the respective networks need to interact with each other, so the ATM signaling protocols need to be modified to understand the existence of mobile users. In Section VI-A, we discuss issues associated with ATM and mobile users.

\section{A. Making ATM Work with Mobile Users: The Issues}

In this section we discuss some of the limitations of the signaling procedures discussed in Section III. We first propose an interim solution and then arrive at a solution which addresses all of the concerns noted here.

The Interim Solution: The ATM signaling protocol currently understands only fixed user numbers. These numbers are treated very much like a phone number to establish where to route a call to any given user. When fixed ATM users are allowed to interact with mobile users we face the following numbering system problem. When an ATM user desires to connect to a user that is a wireless network subscriber, the called user's number will not have meaning to an ordinary ATM host. This implies that we need to have a numbering scheme that accommodates the mobile users. An interim solution is to define PCS subscriber numbers as specially marked ATM numbers ${ }^{12}$ and have ATM to wireless user calls routed to designated wireless user switches that will reroute the calls to their final destinations. A longer-term solution to this problem is given in [21]. The calls from wireless network subscribers to fixed ATM hosts do not constitute a problem as the burden of establishing the connection lies with the wireless network which is aware of both kinds of numbering.

This alternative is easy to implement and does not require any major modification to the ATM protocols. Because of ease of implementation this approach could provide a good interim solution. Unfortunately, as noted earlier, this interim method will not scale well as the number of wireless network users grow and the wireless switching centers get congested. We might also face having to re-route calls across hundreds of miles to establish a connection to a user that is next door. This leads to our next topic which will relieve these problems.

Wireless-Aware ATM Interface Approach: A wirelessaware ATM interface approach will distribute the wireless switching center functionality to all fixed ATM end-points, including ATM switches and ATM interface equipment at user terminals as discussed in Section IV. This will require designating a special numbering scheme which will identify the wireless users as "mobile" and cause a different signaling mechanism to be employed for voice and data calls that are to or from wireless users. These mechanisms will be similar to the wireless network messages discussed in Section IV. For example, for an ATM user ${ }^{13}$ to establish a data connection to a wireless network user, the protocol will recognize that the user is mobile and try to obtain the location information for that user using wireless network signaling procedures. Once the location information is obtained, a connection will be setup. This will distribute the intelligence to the fixed network user interfaces and will result in more effective use of network resources. This approach is incorporated into migratory signaling discussed in Section IV.

\section{B. Wireless Users in a Multitier Environment}

A multitier network environment is defined as one that will provide different bandwidth capacities to users depending on their locality. An example of a multitier architecture is given in [17]. In a campus-wide setting, users may be able to attain a few megabits per second data rates. As they leave the campus and go into a wider area, the wireless may only be able to support a lower data rate, but still may allow the connection to continue in a seamless manner. Accommodating changing data rates on a pre-established connection will require modification to supporting fixed ATM networks. In some environments, a tier change could cause the available channel bandwidth to decrease or to increase by an order of magnitude or more. Clearly, we need to define a procedure by which the bandwidth allocated for an established connection may be changed.

\footnotetext{
${ }^{12}$ By special marking, we mean to assign area codes that will designate the users as mobile and help route the calls to special wireless-to-ATM interface switches.

${ }^{13}$ Note that by an ATM user we mean the physical ATM interface module on a terminal, computer, or a workstation.
} 
TABLE IV

ATM Signaling Message Lengths

\begin{tabular}{l|r|r}
\hline ATM Signaling Message & Minimum Length (bytes) & Maximum Length (bytes) \\
\hline \hline SETUP & 107 & 238 \\
\hline CALL PROCEEDING & 17 & 25 \\
\hline CONNECT & 25 & 53 \\
\hline CONNECT ACK. & 9 & 9 \\
\hline RELEASE & 15 & 43 \\
\hline RELEASE COMPLETE & 13 & 43 \\
\hline
\end{tabular}

One approach to solving this problem can be classified as an extension of ATM flow control algorithms. When the user changes a tier in the multitier network, the ATM flow control mechanisms will either detect an extreme congestion in the case of a bandwidth decrease or they may be programmed to detect a completely empty packet queue in case of a bandwidth increase. This in turn could trigger the network to send a message to either party asking for re-negotiation of ATM traffic descriptors. We shall call this method the Autonomous Bandwidth Negotiation Algorithm.

Alternatively, the wireless access network could notify the ATM network of the tier change. Upon notification the ATM network may change the parameters for the established connection.

\section{Handling Bursts of Errors in the Wireless Network}

The wireless environment suffers from stochastic channel degradation; therefore, occasional bursts of errors may occur for data that is transmitted over a radio interface. In the current ATM adaptation layer implementations, the only recovery from such an error burst is re-transmitting the complete information packet through the network. In order to prevent the excessive use of network resources the wireless network zone managers may need to be able to buffer large amounts of data so that such errors only result in a re-transmission over the radio interface.

\section{Rerouting Strategy for Handoff}

A handoff is performed when a portable unit determines that a better quality radio link is available. Some handoffs result in switching to a neighboring zone. When the handoff process is handled by the network, we may make use of the locality of handoff and choose to re-route at the nearest common network node between the two zones. However, the current ATM signaling does not permit a rerouting at an intermediary point of an ATM connection [6]. A summary of efficient algorithms on rerouting for handoff may be found at [2].

\section{E. Relevant Work on Wireless ATM Networking}

In this section, we briefly mention research related to the topics discussed in this paper (see [13], [14], and [16]). In [19], the author proposes a wireless ATM networking architecture similar to the one discussed here, but considers an overlay network consisting of virtual paths, serving as a signaling backbone network. This paper extends the results given in [19] by providing explicit and detailed procedures for handoff, registration and call setup transactions and presenting a statistical performance analysis of the proposed overlay network architecture. In [20], the authors present a wireless ATM network architectural model, but do not describe explicitly how the support for mobile users will be implemented.

The field of wireless ATM network research has flourished within the past year. In this short section we mentioned research related to that presented in this paper. See the reference list provides a more complete listing of current research on wireless ATM networks.

\section{CONCLUSION}

The integration of the fixed ATM and the next generation wireless personal communications networks into a wireless ATM network is challenging. The current ATM networks do not have the means to understand or to support mobile users. On the other hand, the specifications for current wireless networks are still being designed to interface with the plain old telephone networks. In this paper we proposed two signaling protocol alternatives for wireless ATM networks: an overlay protocol implementation to integrate mobility into the existing ATM protocol stack and a migratory signaling architecture that provided native support for both fixed and mobile ATM users. We also established a database architecture to support mobility in the ATM network. The performance of the overlay and migratory approaches were also analyzed and compared. Finally, we identified issues involved in the interactions between wireless and fixed ATM users, and wireless users in multitier environments.

\section{APPENDIX A \\ ATM Signaling Message Lengths}

The ATM signaling messages mentioned in the text are taken from [6]. According to the specifications, the lengths of ATM signaling messages may vary depending on the situation. We will list the minimum and maximum lengths for these messages in Table IV.

\section{APPENDIX B \\ DeRIVATION OF (1) THROUGH (3)}

In [15], the equations for estimating the mean of database transaction rates are given. We adapted these equations to the wireless ATM network and simplified them. The derivation of these equations are given below.

1) The first equation $L_{c}=N p c(1+q)$ stays the same in both texts.

2) The equation given for registration in [15] is

$$
L_{r}=\frac{(1-s)(1-m)}{\pi}\left(e \rho v P_{p} M j+N_{r} \rho v 4 D\left(1-m_{2}\right)\right) \text {. }
$$


The differences between [15] and this text are as follows:

- $N_{r}$ is equal to one, since the call processing is fully distributed. The value of $m_{2}$ is assumed to be zero for worst case analysis. $\rho$ stands for the population density in a zone.

- Registration transaction does not include the handoff location updates which are accounted for in the handoff transaction, so we omit the e $\rho v P_{p} M j$ part of the equation resulting in $\frac{(1-s)(1-m)}{\pi} N_{r} \rho v 4 D$. Multiply and divide this equation by $D$ and group $\rho D^{2}$ as $N$, the number of people in a zone, and define $T=\frac{D}{v}$. After these modifications we obtain at $\frac{4 N(1-s)(1-m)}{T \pi}$ as given in the text.

3) The equation given in [15] for handoff is $L_{h}=$ $\frac{(1-s)(1-m)}{\pi} e \rho v P_{p} M j$. The product $M j P_{p}$ simplifies to $4 D$. Then if we multiply and divide by $D$ and use $T$ given above we arrive at $\frac{(1-s)(1-m)}{\pi} e 4 \frac{N}{T}$. Note that $P_{p}$ stands for the perimeter of a radio port coverage area and $d$ stands for one side of that perimeter.

\section{REFERENCES}

[1] B. A. Akyol and D. C. Cox, "Handling mobility in a wireless ATM network," presented at INFOCOM'96, San Francisco, CA, 1996.

[2] _ _ "Rerouting for handoff in a wireless ATM network," presented at ICUPC '96, Cambridge, MA, Sept. 28-Oct. 2, 1996.

[3] B. A. Akyol. (1994, November). Modeling of a personal wireless communication network signaling system. Avaliable e-mail: akyol@wireless.stanford.edu.

[4] B. A. Akyol. (1996, October). The overlay signaling approach with permanent virtual circuits. Avaliable e-mail: akyol@ wireless.stanford.edu.

[5] B. A. Akyol and D. C. Cox. (1996, April). the signaling messages of the W-ATM signaling protocol. Available WWW: http://wireless.stanford.edu/ akyol.

[6] ATM Forum User Network Interface Specification Version 3.1, ATM Forum, May 1994.

[7] Q.2931 ATM Network Signaling Specification, ITU.

[8] Redl, H. Siegmund, M. K. Weber, and M. W. Oliphant, An Introduction to GSM. Boston, MA: Artech House, 1995.

[9] EIA/TIA Interim Standard 41. Cellular Radio Telecommunications Intersystem Operations, Washington, DC: Electronic Industries Association, 1991.

[10] D. C. Cox, "Wireless network access for personal communications," IEEE Commun. Mag., vol. 30, no. 12, pp. 96-115, Dec. 1992.

[11] _ "A radio system proposal for widespread low-power tetherless communications," IEEE Trans. Commun., vol. 39, no. 2, pp. 324-335, Feb. 1991.
[12] LATA Switching Systems Generic Requirements, Bellcore Technical Reference TR-TSY-000517, Issue 3, Mar. 1989, Sec. 17, Table 17.6-B: "Traffic Capacity and Environment."

[13] R. R. Gejji, "Mobile multimedia scenario using ATM and microcellular technologies," IEEE Trans. Veh. Technol., vol. 43, no. 3, pp. 699-703, Aug. 1994.

[14] I. M. Leslie et al., "ATM everywhere," IEEE Network, pp. 40-46, Mar. 1993.

[15] C. N. Lo et al., "An estimate of network database transaction volume to support universal PCS," in ICUPC '92, Dallas, TX, Sept. 29-Oct. $1,1992$.

[16] A. Miller, "From here to ATM," IEEE Spectrum, pp. 20-24, June 1994

[17] J. F. Rizzo and N. R. Sollenberger, "Multitier wireless access," IEEE Personal Commun. Mag., June 1995.

[18] A. S. Acampora, "An architecture and methodology for mobile-executed handoff in cellular ATM networks," IEEE J. Select. Areas Commun., vol. 12, no. 8, pp. $1365-1375$, Oct. 1994.

[19] B. Rajagopalan, "Mobility management in integrated wireless ATM networks," in MOBICOM'95, Berkeley, CA, Nov. 1995.

[20] M. Barton and T. R. Hsing, "Architecture for wireless ATM networks," in PIMRC '95, Toronto, Canada, Sept. 27-29, 1995.

[21] R. Jain, S. Rajagopalan, and L. F. Chang, "Phone number portability for PCS systems with ATM backbones using distributed dynamic hashing," IEEE J. Select. Areas Commun., submitted for publication.

[22] D. Raychaudhuri et al., "ATM based transport architecture for multiservices wireless personal communication networks," IEEE J. Select. Areas Commun., pp. 1401-1414, Oct. 1994.

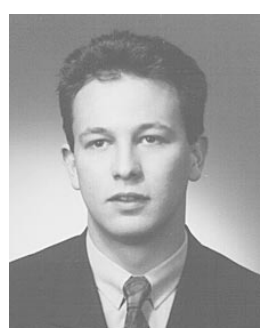

Bora A. Akyol (S'92) received the B.S. degree from Bilkent University, Ankara, Turkey, in 1992, and the M.S. degree from Stanford University, Stanford, CA, in 1993, both in electrical engineering.

Currently, he is a Ph.D. candidate at Stanford University, Stanford, CA, researching signaling and media-access protocols for wireless ATM networks.

Donald C. Cox (S'58-M'61-SM'72-F'79) for a photograph and biography, see this issue, p. 3. 